Background The objective of this research is to investigate Greek drivers willingness to pay for innovative vehicle insurance schemes such as Pay-as-you-drive (PAYD) and Pay-how-you-drive (PHYD) schemes. Current technological advances, enable to collect high resolution driver behaviour data easier and more accurately using technologies such as smartphones, On-Board Diagnostics (OBD) systems etc. and use them to monitor, analyse and evaluate each individual driver's behaviour. PAYD scheme is based on assessing driver's level of exposure to traffic risk while PHYD is based on driver behaviour indicators such as speeding, acceleration etc. The implementation of this policy has shown a significant impact on driver's behaviour and as a result, this measurement is a matter of great importance for traffic safety.

Methods Within this research, a stated-preference survey is designed and administered in a questionnaire form both paperbased and online. The questionnaire is comprised of four sections including a general driving information, a driving behaviour information, a new innovative insurance policy scenario and a demographic characteristics section. At the third section, respondents are given several scenarios to choose between the standard and the new insurance policy in order to measure the sensitivity of their choice based on some factors such as the variation of mileage, the average speed and the annual insurance cost. Subsequently, a discrete choice model was developed to analyse data collected during this experiment.

Results indicate a strong correlation between policy choice and annual cost variation.

Conclusions Willingness to switch to a new innovative policy was found statistically significant especially for a cost reduction of $20 \%$. Finally, mileage and average speed variation was found to affect people's choice less than the innovative policy's cost but still statistically significant.

\section{RISK FACTORS FOR BICYCLING INJURIES IN CHILDREN AND ADOLESCENTS: A SYSTEMATIC REVIEW}

${ }^{1}$ Tania Embree, ${ }^{1,2}$ Nicole Romanow, ${ }^{1}$ Maya Djerboua, ${ }^{3}$ Natalie Morgunov, ${ }^{3}$ Jacqueline Williamson, ${ }^{1}$ Brent Hagel. 'Department of Paediatrics, University of Calgary, Canada; ${ }^{2}$ Faculty of Kinesiology, University of Calgary, Canada; ${ }^{3}$ Cumming School of Medicine, University of Calgary, Canada

\subsection{6/injuryprev-2016-042156.901}

Background Bicycling injuries in young people represent a substantial cost to health care systems. The objective of this review was to examine the individual and environmental factors associated with bicycling-related injury risk in children and youth.

Methods Fourteen electronic databases were searched using exploded subject headings and keywords. Two authors independently screened article titles and abstracts for inclusion. The fulltext of the potential articles was assessed to determine eligibility. The inclusion criteria were bicyclists less than 18 years old; individual and environmental characteristics of bicycling outcomes; comparisons between injured and uninjured bicyclists, injury type or severity level; study designs with a pre-determined comparison group; and publications in English from 1990 to May 2015. The exclusion criteria were injury outcomes related to helmet use, helmet legislation or mountain biking; comparisons of censusbased injury rates; cross-sectional studies; and letters to the editor. A modified version of the Newcastle-Ottawa Scale was used to assess study quality.
Results Fifteen articles met the inclusion and exclusion criteria. Overall, 46 different risk factors were examined. The most commonly reported risk factors were age ( $\mathrm{N}=10$ studies), sex $(\mathrm{N}=7)$, equipment related factors $(\mathrm{N}=6)$, bicycling exposure $(\mathrm{N}=5)$, bicycling purpose $(\mathrm{N}=5)$, and motor vehicle $(\mathrm{MV})$ collision $(\mathrm{N}=4)$. The reviewed studies varied in quality; the main weaknesses were inadequate definitions of study groups, lack of control for potential confounders, and the use of self-reported data.

Conclusions While many of the studies had significant limitations, one recurring theme was that young bicyclists received more severe injuries when exposed to MV collisions. To reduce injuries in children and adolescents, we recommend separating bicyclists from MVs on the road and implementing strategies to reduce traffic speed and volume.

\section{PREVALENCE AND FACTORS ASSOCIATED WITH ROAD TRAFFIC CRASH AMONG TAXI DRIVERS IN MEKELLE TOWN, NORTHERN ETHIOPIA, 2014: A CROSS SECTIONAL STUDY}

${ }^{1}$ Nigus Gebremedhin Asefa, ${ }^{2}$ Lalit Ingale , ${ }^{2}$ Ashenafi Shumey $\sharp{ }^{3}$ Hannah Yang $\ddagger .{ }^{1}$ Tewelde Legesse Health Sciences College, Mekelle, Tigray, Ethiopia; ${ }^{2}$ Mekelle University, College of Health Sciences, Mekelle, Tigray, Ethiopia; ${ }^{3}$ Department of International Health, Johns Hopkins Bloomberg, School of Public Health, Baltimore, Maryland, USA; $¥$ These Authors Contributed Equally to This Work

\subsection{6/injuryprev-2016-042156.902}

Objectives The 2013 World Health Organisation Status Report on Road Safety estimated that approximately 1.24 million deaths occur annually due to road traffic crashes with most of the burden falling on low- and middle-income countries. The objective of this research is to study the prevalence of road traffic crashes in Mekelle, Tigray, Northern Ethiopia and to identify risk factors with the ultimate goal of informing prevention activities and policies.

Methods This study used a cross-sectional design to measure the prevalence and factors associated with road traffic crashes among 4-wheeled minibus $(\mathrm{n}=130)$ and 3-wheeled Bajaj $(\mathrm{n}=582)$ taxi drivers in Mekelle, Ethiopia. Bivariate and multivariate logistic regression were used to evaluate the association between risk factors and drivers' involvement in a road traffic crash within the 3 years prior to the survey.

Results Among the 712 taxi drivers, 26.4\% ( $n=188$ ) of them reported involvement in a road traffic crash within the past 3 years. Drivers who listened to mass media had decreased likelihood of road traffic crash involvement (Adjusted odds ratio, AOR: $=0.51,0.33-0.78$ ), while speedy driving (AOR: $=4.57$, $3.05-7.44$ ), receipt of a prior traffic punishment (AOR: $=4.57$, 2.67-7.85), and driving a mechanically faulty taxi (AOR: $=4.91$, 2.81-8.61) were strongly associated with road traffic crash involvement. Receiving mobile phone calls while driving (AOR: $=1.91,1.24-2.92)$ and history of alcohol use (AOR: $=1.51,1.00-2.28)$ were also associated with higher odds of road traffic crash involvement.

Conclusion The results of this study show that taxi drivers in Mekelle habitually place themselves at increased risk of road traffic crashes by violating traffic laws, especially related to speedy driving, mobile phone use, and taxi maintenance. This research can be used to support reevaluation of the type, severity, and enforcement of traffic violation penalties. 